\title{
Acoustic topological insulator and robust one-way sound transport
}

\author{
Cheng He ${ }^{1,2}, \mathrm{Xu} \mathrm{Ni}^{1}, \mathrm{Hao} \mathrm{Ge}^{1}$, Xiao-Chen Sun${ }^{1}$, Yan-Bin Chen ${ }^{1}$, Ming-Hui Lu ${ }^{1,2 \star}$, Xiao-Ping Liu ${ }^{1,2 \star}$ \\ and Yan-Feng Chen ${ }^{1,2 \star}$
}

\begin{abstract}
Topological design of materials enables topological symmetries and facilitates unique backscattering-immune wave transport ${ }^{1-26}$. In airborne acoustics, however, the intrinsic longitudinal nature of sound polarization makes the use of the conventional spin-orbital interaction mechanism impossible for achieving band inversion. The topological gauge flux is then typically introduced with a moving background in theoretical models ${ }^{19-22}$. Its practical implementation is a serious challenge, though, due to inherent dynamic instabilities and noise. Here we realize the inversion of acoustic energy bands at a double Dirac cone ${ }^{15,27,28}$ and provide an experimental demonstration of an acoustic topological insulator. By manipulating the hopping interaction of neighbouring 'atoms' in this new topological material, we successfully demonstrate the acoustic quantum spin Hall effect, characterized by robust pseudospin-dependent one-way edge sound transport. Our results are promising for the exploration of new routes for experimentally studying topological phenomena and related applications, for example, sound-noise reduction.
\end{abstract}

The topological insulator, characterized by the quantum spin Hall effect (QSHE), originates from condensed matter. A prerequisite condition for such an effect, Kramers doublet, can be fulfilled thanks to the intrinsic spin-1/2 fermionic character of electrons. Thus, the key physics behind realizing a bosonic (for example, photonic or phononic) analogue of the electronic topological insulator is to increase the degrees of freedom so as to create a double Dirac cone, where Kramers doublet exists in the form of two two-fold states (pseudospin-up and pseudospin-down). For example, a photonic topological insulator often takes advantage of two polarizations of a spin-1 photon, which are utilized to construct the required Kramers doublet, for example, degenerate $\mathrm{TM}+\mathrm{TE} / \mathrm{TM}-\mathrm{TE}$ states ${ }^{11}$ (TM and TE represent transverse magnetic and electric polarizations, respectively), TM/TE states ${ }^{29}$, or left/right circularly polarized states ${ }^{30}$. An acoustic system especially for longitudinal airborne sound, however, possesses intrinsic spin-0, giving rise to no such polarization-based Kramers doublet and thus hindering acoustic QSHE. To overcome this issue, Kramers doublet in the form of degenerate artificial acoustic spin-1/2 states may be constructed, for example, using clockwise and anticlockwise circulating acoustic waves ${ }^{23}$. Spin-orbital interaction may then be introduced in a time-dependent fashion ${ }^{24}$. Most recently, ref. 15 proposed a novel concept for realizing a photonic topological insulator by utilizing two pairs of degenerate Bloch modes of TM polarization (instead of two polarizations) as a result of the zone folding mechanism in a composite lattice structure ${ }^{15}$. Here, we show, other than the zone folding mechanism ${ }^{15}$, a double cone can also be accidentally formed by deliberately manipulating the filling factor of a honeycomb lattice. This straightforward yet elegant approach takes advantage of acoustic systems' large index and impedance contrast, which unfortunately is usually absent in photonic systems (see Supplementary Information). On the basis of our novel concept, we experimentally demonstrate a completely passive or static two-dimensional acoustic topological insulator, utilizing phononic 'graphene' consisting of stainless-steel rods in air.

In our phononic 'graphene', an artificial spin-1/2 is emulated through mode hybridization near the dispersion degeneracy of different energy bands. Specifically, two degenerate modes $M_{1}$ and $M_{2}$ at a given frequency can be hybridized to construct two new superimposed degenerate spin-1/2 states: spin $\pm \equiv M_{1}+i M_{2} / M_{1}-i M_{2}$. Since in this scenario each spin-1/2 state corresponds to two degenerate modes, the two-fold degeneracy of the Dirac cone in a spin-1/2 electronic system, where the QSHE occurs, is replaced with a four-fold degeneracy or a double Dirac cone. By engineering the nearest neighbour coupling in a phononic 'graphene' with $C_{6 \mathrm{v}}$ symmetry, an accidental double Dirac cone can be realized ${ }^{27,28}$ (see Supplementary Information), because the $C_{6 \mathrm{v}}$ symmetry, according to group theory, possesses two two-dimensional irreducible representations, which can be leveraged to construct a double Dirac cone by forming a four-fold accidental degeneracy ${ }^{31}$. Note that similar double Dirac cones have also been reported previously in other classic wave systems, such as electromagnetic waves ${ }^{15,31,32}$ and elastic waves ${ }^{18}$. Different from all of these works $s^{27,28}$, we show that, in addition to the double Dirac cone, the phononic 'graphene' studied here undergoes a symmetry inversion in reciprocal space, causing energy band inversion, which ultimately leads to a distinct topological phase transition ${ }^{15}$.

The topological phase transition can be leveraged to form topologically protected gapless edge states inside the bulk frequency bandgap at the interface between two phononic crystals with topologically dissimilar phases, which can then be exploited to construct a topologically protected waveguide as shown in Fig. 1a. Due to topological protection, the acoustic backscattering can be largely suppressed and thus robust against various kinds of defect (including cavities, disorders and even sharp bends). To identify these topological phases, the evolution of energy band structures and their acoustic states with decreasing filling ratio are illustrated in Fig. 1b. At a high filling ratio, for example, $r / a=$ 0.45 (left panel of Fig. 1b), separated by a bandgap, two two-fold

\footnotetext{
${ }^{1}$ National Laboratory of Solid State Microstructures \& Department of Materials Science and Engineering, Nanjing University, Nanjing, Jiangsu 210093, China. ${ }^{2}$ Collaborative Innovation Center of Advanced Microstructures, Nanjing University, Nanjing, Jiangsu 210093, China. *e-mail: luminghui@nju.edu.cn; xpliu@nju.edu.cn; yfchen@nju.edu.cn
} 
a

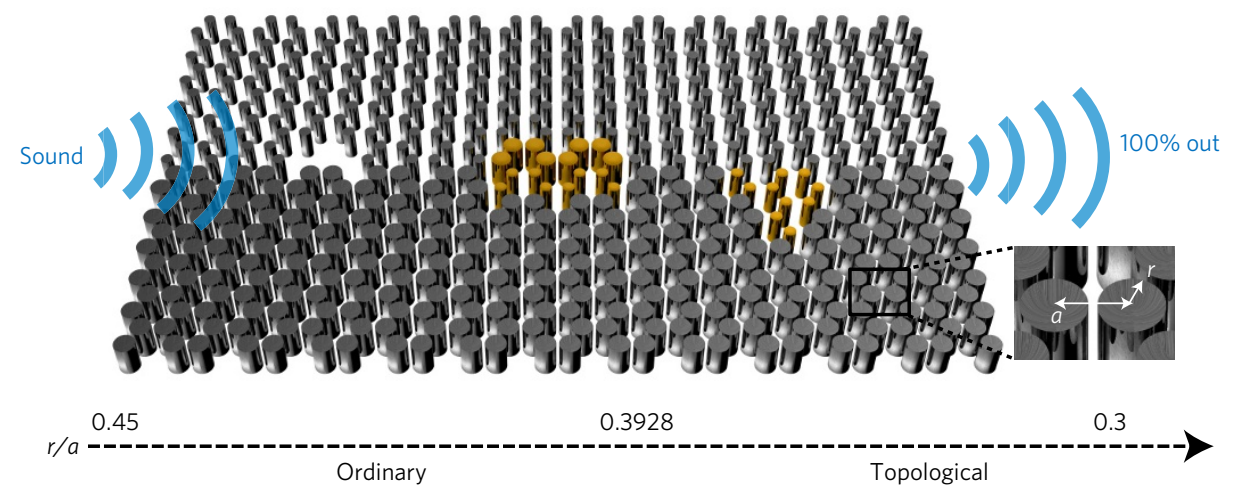

b
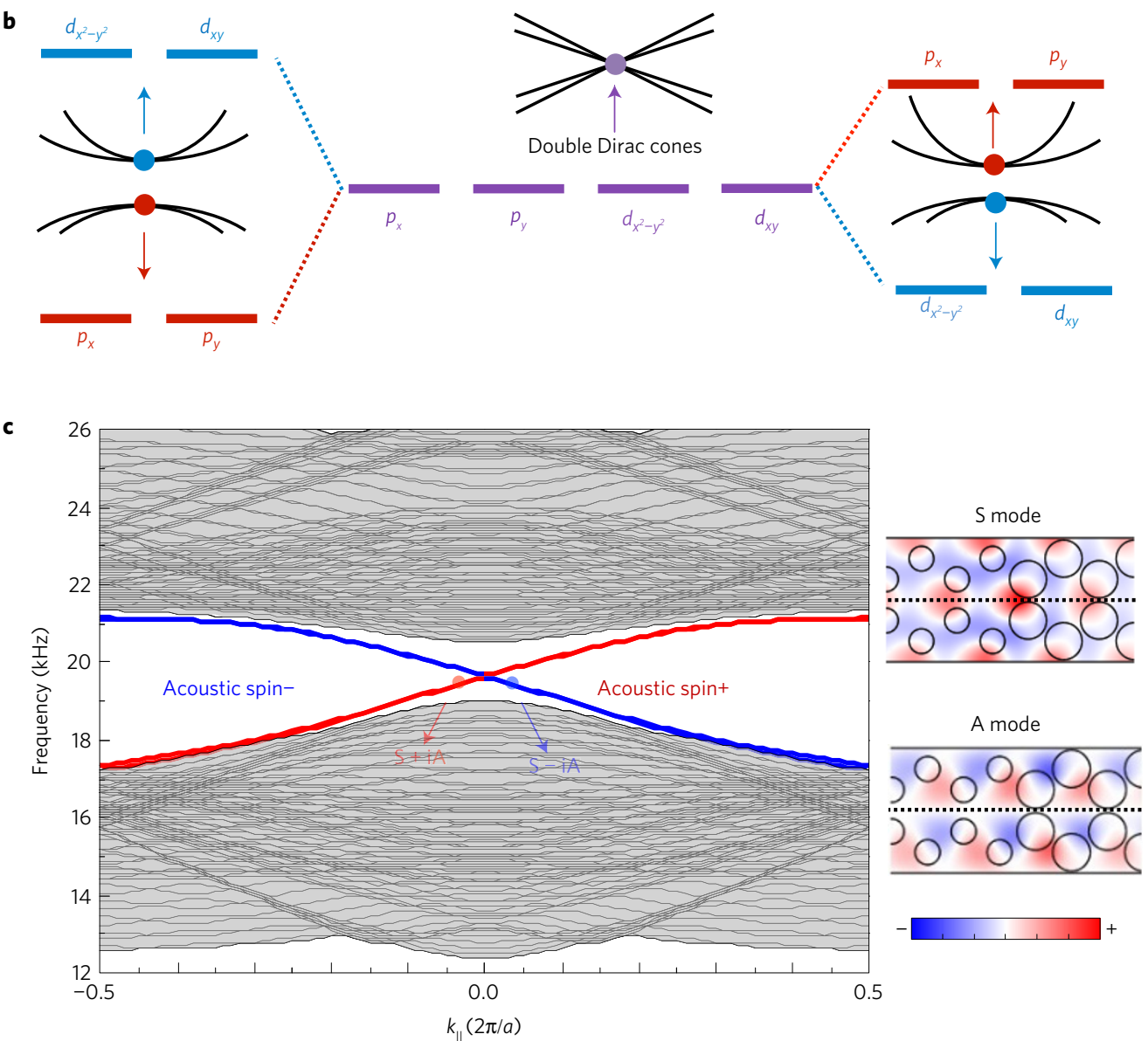

Figure 1 | Schematic of the acoustic insulator and band inversion process. a, A schematic of our proposed acoustic topological insulator constructed with two types of phononic 'graphene' with the same lattice constant $a$ but different 'atom' (stainless-steel rod) radii $r$. This novel design enables one-way robust spin-dependent transportation against defects such as a cavity, a lattice disorder and a bend as indicated in the figure. The inset shows a zoom-in view of our phononic 'graphene'. $\mathbf{b}$, Illustration of a band inversion process with a decreasing filling ratio. At a large filling ratio, for example, $r / a=0.45$, the phononic crystal has two two-fold degenerate acoustic states denoted by $p_{x} / p_{y}$ and $d_{x^{2}}-y^{2} / d_{x y}$, which are separated by a bulk bandgap; at a decreased filling ratio, for example, $r / a=0.3928$ in our case, the bulk bandgap disappears and an accidental double Dirac cone with a four-fold degeneracy is formed; at an even smaller filling ratio, for example, $r / a=0.3$, the bulk bandgap reappears along with two inverted two-fold degenerate acoustic states,

corresponding to $d_{x^{2}-y^{2}} / d_{x y}$ and $p_{x} / p_{y}$. In this process, a topological phase transition occurs near the double Dirac cone. Note sketches of energy bands are shown as black curves. c, Calculated projected energy bands of a supercell consisting of a topological phononic crystal $(r=0.3 \mathrm{~cm}, a=1 \mathrm{~cm})$ stacked with an ordinary phononic crystal $(r=0.45 \mathrm{~cm}, a=1 \mathrm{~cm})$. The material parameters used for calculation are the density and longitudinal sound speed of $7,800 \mathrm{~kg} \mathrm{~m}^{-3}$ and $6,010 \mathrm{~m} \mathrm{~s}^{-1}$ for stainless steel and $1.25 \mathrm{~kg} \mathrm{~m}^{-3}$ and $343 \mathrm{~m} \mathrm{~s}^{-1}$. The red and blue lines represent an acoustic spin + and spin- edge state that is hybridized with a symmetric edge mode $(\mathrm{S})$ and anti-symmetric edge mode $(\mathrm{A})$ based on $\mathrm{S}+\mathrm{i} A$ and $\mathrm{S}-\mathrm{i} \mathrm{A}$, respectively. The right panel shows a representative example of the pressure field distribution at $k_{\|}=0.05$ for the $S$ and $A$ modes. The shadow regions represent the bulk energy bands.

degeneracy, one for the lower bands $p_{x} / p_{y}$ and the other for the upper bands $d_{x^{2}-y^{2}} / d_{x y}$, appears at the Brillouin zone centre. Spin$1 / 2$ for the bulk states can thus be achieved through hybridizing these $p / d$ states as $p_{ \pm}=\left(p_{x} \pm i p_{y}\right) / \sqrt{2}$ and $d_{ \pm}=\left(d_{x^{2}-y^{2}} \pm i d_{x y}\right) / \sqrt{2}$ (ref. 15). Similar to $p$ and $d$ orbitals of electrons, here $p_{x}$ obeys symmetry $\sigma_{x} / \sigma_{y}=-1 /+1 ; p_{y}$ obeys $\sigma_{x} / \sigma_{y}=+1 /-1 ; d_{x^{2}-y^{2}}$ obeys $\sigma_{x} / \sigma_{y}=+1 /+1$; and $d_{x y}$ obeys $\sigma_{x} / \sigma_{y}=-1 /-1$, where $\sigma_{x, y}=+1,-1$ means the even or odd symmetry along the $x$ or $y$ axis of the 
a
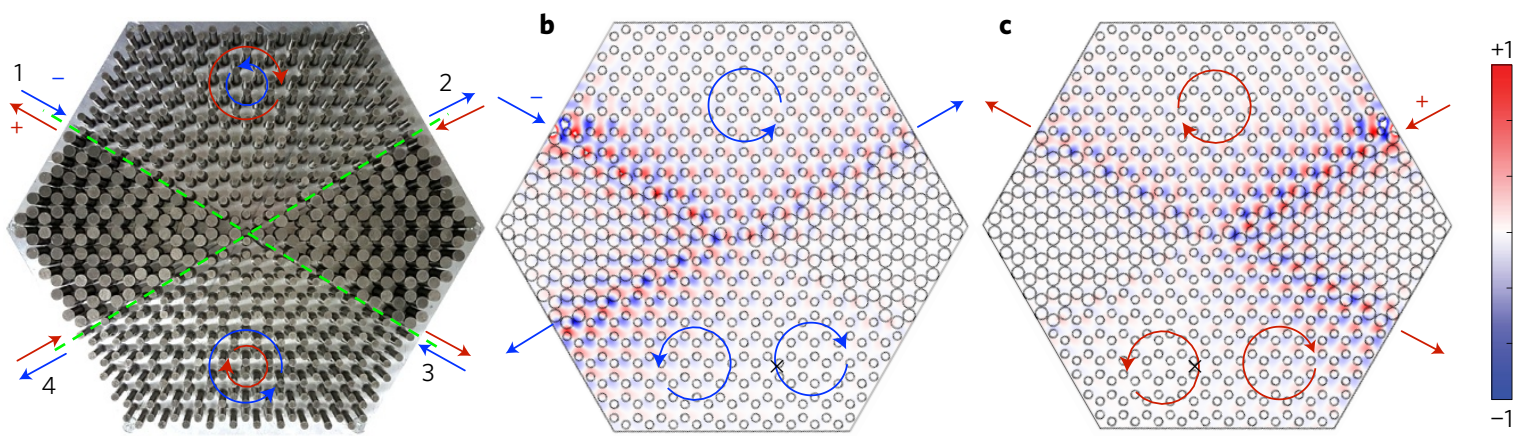

d

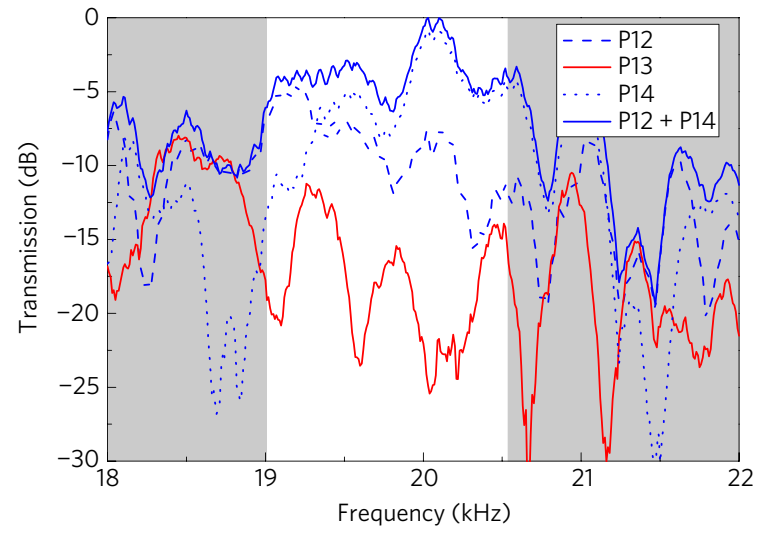

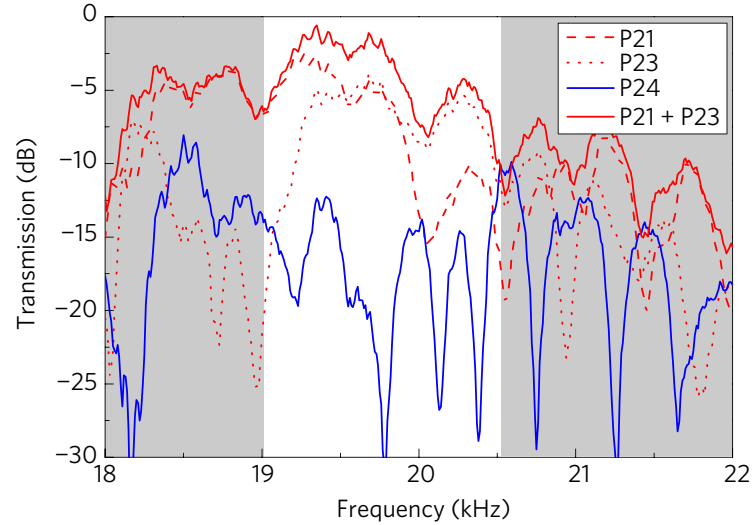

Figure 2 | Acoustic one-way spin-dependent transport. a, A photo of the cross-waveguide splitter sample used in our experiment. Topological phononic

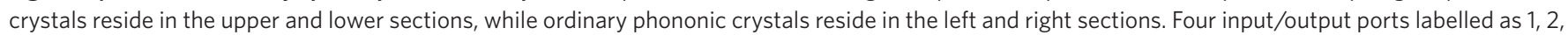

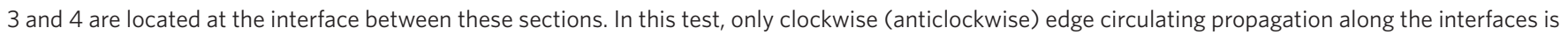
allowed for acoustic spin+ (spin-) as indicated by the red (blue) circular arrow. b,c, Simulated acoustic pressure field distribution at a frequency of

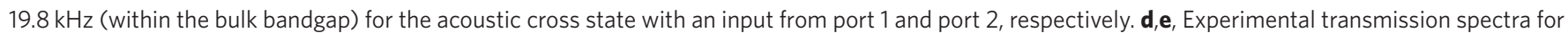
acoustic spin- incidence from port 1 and for acoustic spin+ incidence from port 2, respectively. Pij represents the acoustic transmission spectrum from port $i$ to port $j(i=1,2$ and $j=1,2,3,4)$ including the in and out coupling losses. The shadow regions indicate the bulk energy bands.

unit cell, respectively (see Supplementary Information). With a deceased filling ratio of $r / a=0.3928$ (middle panel of Fig. 1b), the energy bandgap is eliminated, resulting in an accidental double Dirac cone with the desired four-fold degeneracy at the Dirac point. Further decreasing the filling ratio, for example, $r / a=0.3$, destroys the four-fold degeneracy, and reopens the bandgap (right panel of Fig. 1b). In this process, the symmetry in reciprocal space is inverted, causing the energy band inversion, which is confirmed by examining the Bloch modes before the bandgap disappears and after it reappears (see Supplementary Information). The acoustic band inversion effect here is related to the fact that the velocity of a longitudinal acoustic wave in an air background is much slower than that in stainless-steel rods. The created symmetry inversion in phononic 'graphene' further leads to a topological transition from an ordinary phononic crystal (OPC) to a topological phononic crystal (TPC) (see Supplementary Figs 1 and 2 for details). Note that the acoustic topological insulator we proposed here is based on an accidental double Dirac cone resulting from $C_{6 \mathrm{~V}}$ symmetry. In principle, other configurations, as long as they comply with the required $C_{6 \mathrm{~V}}$ symmetry, can have the same phenomenon, for example, the ring-shaped components in triangle lattices ${ }^{28,32}$.

The projected band structure of a TPC-OPC supercell is shown in Fig. 1c. Inside the overlapped bulk energy bandgap of the two phononic crystals, it is evident that there exists a pair of edge states (red and blue lines). These edge states are localized at the interface between the TPC and the OPC and support edge propagation of sound. Acoustic spin \pm of the edge states are obtained via hybridizing a symmetric mode (S) and an anti-symmetric mode (A) shown in the right panel of Fig. 1c as $\mathrm{S}+\mathrm{iA} / \mathrm{S}-\mathrm{iA}$, respectively. They can also be correlated to the spins of the bulk states in the TPC and the OPC located on either side of the interface as: $\mathrm{S}+\mathrm{iA} / \mathrm{S}-\mathrm{iA} \equiv p_{+}+d_{+} / p_{-}+d_{-}$(see Supplementary Information). Hence, the symmetric component $S$ can be represented as $\mathrm{S}=\left(p_{x}+d_{x^{2}-y^{2}}\right) / \sqrt{2}$, while the anti-symmetric component $\mathrm{A}$ as $\mathrm{A}=\left(p_{y}+d_{x y}\right) / \sqrt{2}$. The two acoustic spin edge states have the same sound velocity amplitude but in opposite directions, implying the existence of spin-orbital coupling and thus one-way spin-dependent propagation.

Generally, it is very difficult to selectively excite a particular pseudospin in experiment even with multiple excitation sources. Here, we utilize a cross-waveguide splitter ${ }^{33}$, which allows us to study pseudospin-dependent transport with a very high fidelity even in the case of unknown pseudospin excitation state. As shown in Fig. 2a, the splitter is divided into four sections with the TPC residing in the upper and lower sections and the OPC residing in the left and right sections. There are four input/output ports in this splitter (labelled as 1, 2, 3 and 4). For an acoustic bar state, that is, the sound transmitting straight through from port 1 to port 3 (or from port 2 to port 4), with respect to its transmission direction, the TPC is located on the left side while the OPC is located on the right side before the acoustic wave propagates across the central region of the splitter. However, such kind of structural spatial symmetry is inverted immediately after the acoustic wave propagates across the centre (OPC on the left side and TPC on the right side). Since either pseudospin state is preserved across the centre region, the edge state is not allowed to propagate in a straight through fashion, 


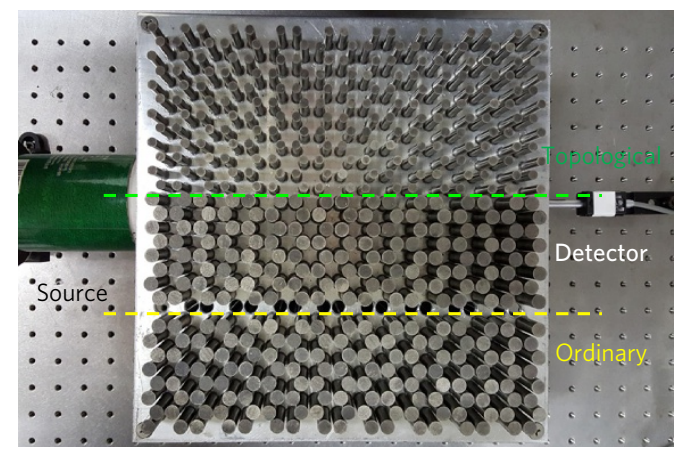

b

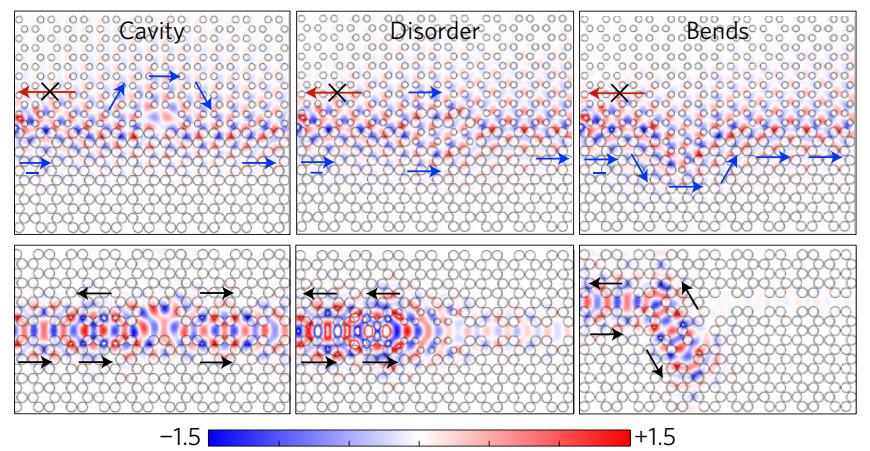

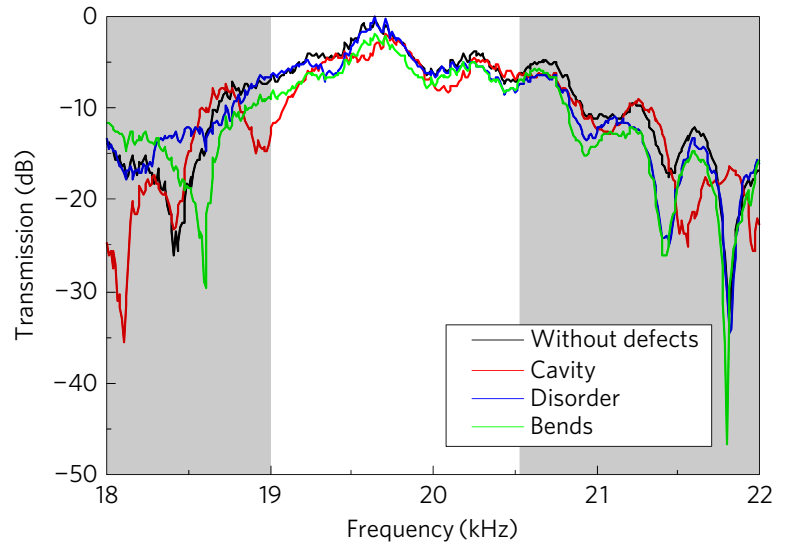

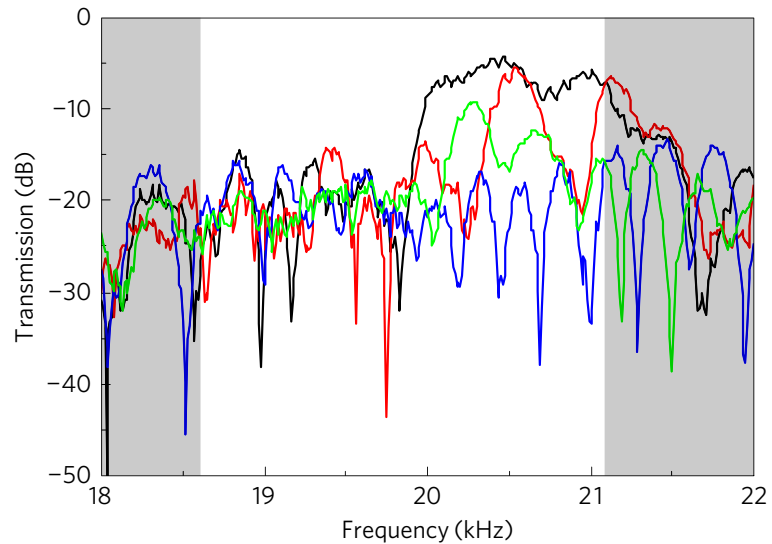

Figure 3 | Robust one-way sound transport. a, A photo of our experimental set-up. The green and yellow dashed lines indicate the location of an acoustic topological waveguide and an ordinary bandgap-guiding phononic crystal waveguide, respectively. $\mathbf{b}$, Simulated acoustic pressure field distribution for acoustic spin - incident at $20.1 \mathrm{kHz}$ (within the bulk bandgap) in three different configurations, corresponding to three different defects, that is, a cavity, a lattice disorder and a bend. Blue, red and black arrows denote the propagation direction of acoustic spin-, spin + and ordinary sound, respectively. c,d, Experimental transmission spectra for the topological waveguide and ordinary bandgap-guiding phononic crystal waveguide, respectively. The black curve corresponds to the case without any defects, while the red, blue and green curves indicate the transmission spectrum for the case with a cavity, a lattice disorder and a bend, respectively. The shadow regions correspond to the bulk energy bands.

implying that the acoustic bar state in this splitter is completely prohibited for all pseudospin edge states. Similar analysis can show that for an acoustic cross state, for example, from port 1 to port 2 or to port 4 , the structural spatial symmetry is always preserved and the propagation of edge states is always allowed. In addition, the acoustic cross state is spin-dependent, which is determined by the spatial symmetry. For instance, in Fig. 2a, the acoustic cross state with input sound at port 1 or at port 3 supports only the spin+ edge state while the cross state for the other two ports supports the spin- edge state. This unique effect can be explained by checking the slope of the dispersion band, that is, the group velocity, for each individual acoustic spin edge state in Fig. 1c. Clearly, because of their opposite slope across the whole Brillouin zone, the acoustic spin + edge state and spin- edge state can travel only in a one-way fashion but with opposite directions, clockwise (red circular arrows) versus anticlockwise (blue circular arrows) as illustrated in Fig. 2a. Such a transport behaviour characterizes an acoustic counterpart of the QSHE.

Figure $2 \mathrm{~b}, \mathrm{c}$ shows the simulated pressure field distribution with inputs at port 1 and at port 2 respectively for an acoustic frequency in the bulk bandgap, which resemble clearly two cross states with almost no transmission into the through port in both cases. Such an observation is consistent with our theoretical analysis above. These configurations are then experimentally studied with the resulting transmission spectra shown in Fig. 2d,e. It is found that the acoustic bar state is heavily suppressed as evidently observed from its low transmission, $<-10 \mathrm{~dB}$ for the through port. The spin-dependent cross state for the two acoustic spins, however, remains with very high transmission for the two cross output ports. These experimental observations for the cross-waveguide splitter model confirmed clearly the unique one-way spin-dependent transportation in our acoustic topological insulator.

The demonstrated topological edge states are also symmetry protected with topological immunity against all non-spin-mixing defects. Different defects are intentionally introduced into our topological waveguide near the TPC/OPC interface to study the propagation robustness of our acoustic topological edge states. In comparison, a bandgap-guiding phononic crystal waveguide is also constructed with two OPCs and similar defects are introduced for a control experiment. A picture of the test sample is shown in Fig. 3a, where the location of the topological waveguide and the phononic crystal waveguide can be seen. Three different defects, including a cavity, a lattice disorder, and a bend, are implemented in the two waveguides. Note that all these three types of defect are none-spin-mixing defects by their nature, meaning that no effective 'magnetic' impurities are presented intentionally to break the topological characteristics ${ }^{15,16,18,30,34}$. Simulation results show that with a spin- acoustic wave incidence from the left the edge state in the topological waveguide can detour around all these defects and maintain a very high transmission (three upper panels of Fig. 3b). In contrast, the results for the bandgap-guiding phononic crystal waveguide are drastically different. The cavity in this case causes acoustic resonances, while the disorder and bend severely inhibit the acoustic forward propagation, leading to a decreased 
transmission or even a total reflection (three lower panels of Fig. 3b). The experimentally measured transmission spectra for these two waveguides are shown in Fig. $3 \mathrm{~d}$. The transmission through the topological waveguide is nearly unaffected by any of these defects especially for the spectral region near the centre of the bulk bandgap. However, their presence causes significant disturbance on the sound transportation in the bandgap-guiding phononic crystal waveguide, which could include largely reduced transmission especially on the high-frequency side of the bandgap and multiple resonance dips corresponding to the cavity resonances. Hence, the topological waveguide clearly distinguishes itself from the bandgap-guiding phononic crystal waveguide with the unparalleled advantage of robust sound transportation.

In our acoustic system, the topological property of the TPC is characterized by a non-zero spin Chern number of bulk bands (see Supplementary Information), implying that the edge states are preserved under continuous deformations. Note that a tiny gap exists between two dispersions of the edge states at the $k_{\|}=0$ point (see Supplementary Information), which is originated from an imperfect cladding layer rather than the TPC itself. For instance, in our case we use the topologically trivial phononic crystal as a cladding layer but its eigen states are slightly different from those of the TPC, which gives rise to a theoretically calculated absolute bandgap of $0.095 \mathrm{kHz}$ or a relative bandgap less than $0.5 \%$. Such a bandgap can hardly be probed in our swept frequency tests (Figs $2 \mathrm{~d}$,e and $3 \mathrm{c}, \mathrm{d}$ ) even with a frequency increment of $0.01 \mathrm{kHz}$ because of the usual finite size issue for fabricated samples and the propagation loss for airborne sound. If desired, this relative bandgap can be further reduced to less than $0.02 \%$ in theory by adjusting the boundary condition ${ }^{35}$, that is, changing the filling factor of a row of cylinders to be $r / a=0.1$ (see Supplementary Information). In principle, this tiny bandgap can even be closed with a deliberately designed cladding layer, if it can support the same eigen states as those of the TPC. In reality, any fabrication imperfection could lead to a tiny gap. In this regard, the backscattering is not completely forbidden in experiment. However, the real issue is whether the experimental measurement can detect such a tiny bandgap. What we have found in our work is that the transmission through our topological waveguide is nearly unaffected (Figs 2d,e and $3 c, d)$, suggesting that the bandgap is hardly noticeable and the backscattering is largely suppressed.

The acoustic topological states obtained here pave a new way for studying topological properties in classic wave systems without any noises resulting from dynamical modulation. This unique topological material can in principle be leveraged to explore quantum behaviour of classical waves at a high fidelity. In addition, our design principle and measurement technique demonstrated here can be easily extended to cover a large acoustic spectrum from audible sound to ultrasonic frequencies, and they can even be extended to other classic waves such as Lamb waves. Moreover, our demonstrated topological phenomenon of sound may generate considerable impact in applications in the foreseeable future. For instance, when combined with a low propagation loss substrate/background environment, the backscattering-immune sound transportation may give rise to an ultrahigh-Q acoustic resonance, which, to date, remains a challenge in the acoustic industry.

\section{Methods}

Methods, including statements of data availability and any associated accession codes and references, are available in the online version of this paper.

Received 28 December 2015; accepted 28 July 2016; published online 29 August 2016

\section{References}

1. Klitzing, K. V., Dorda, G. \& Pepper, M. New method for high-accuracy determination of the fine-structure constant based on quantized Hall resistance. Phys. Rev. Lett. 45, 494-497 (1980).

2. Thouless, D. J., Kohmoto, M., Nightingale, M. P. \& den Nijs, M. Quantized Hall conductance in a two-dimensional periodic potential. Phys. Rev. Lett. 49, 405-408 (1982)

3. Hasan, M. Z. \& Kane, C. L. Colloquium: topological insulators. Rev. Mod. Phys. 82, 3045-3067 (2010)

4. Qi, X.-L. \& Zhang, S.-C. Topological insulators and superconductors. Rev. Mod. Phys. 83, 1057-1110 (2011).

5. Haldane, F. D. M. \& Raghu, S. Possible realization of directional optical waveguides in photonic crystals with broken time-reversal symmetry. Phys. Rev. Lett. 100, 013904 (2008).

6. Wang, Z., Chong, Y., Joannopoulos, J. D. \& Soljacic, M. Observation of unidirectional backscattering-immune topological electromagnetic states. Nature 461, 772-775 (2009).

7. Lu, L., Joannopoulos, J. D. \& Soljacic, M. Topological photonics. Nat. Photon. 8, 821-829 (2014).

8. Hafezi, M., Demler, E. A., Lukin, M. D. \& Taylor, J. M. Robust optical delay lines with topological protection. Nat. Phys. 7, 907-912 (2011).

9. Hafezi, M., Mittal, S., Fan, J., Migdall, A. \& Taylor, J. M. Imaging topological edge states in silicon photonics. Nat. Photon. 7, 1001-1005 (2013).

10. Rechtsman, M. C. et al. Photonic floquet topological insulators. Nature 496, 196-200 (2013)

11. Khanikaev, A. B. et al. Photonic topological insulators. Nat. Mater. 12, 233-239 (2013)

12. Chen, W.-J. et al. Experimental realization of photonic topological insulator in a uniaxial metacrystal waveguide. Nat. Commun. 5, 5782 (2014).

13. Lu, L. et al. Experimental observation of Weyl points. Science 349, 622-624 (2015)

14. Bliokh, K. Y., Smirnova, D. \& Nori, F. Quantum spin Hall effect of light. Science 348, 1448-1451 (2015).

15. Wu, L.-H. \& Hu, X. Scheme for achieving a topological photonic crystal by using dielectric material. Phys. Rev. Lett. 114, 223901 (2015).

16. Süsstrunk, R. \& Huber, S. D. Observation of phononic helical edge states in a mechanical topological insulator. Science 349, 47-50 (2015).

17. Wang, P., Lu, L. \& Bertoldi, K. Topological phononic crystals with one-way elastic edge waves. Phys. Rev. Lett. 115, 104302 (2015).

18. Mousavi, S. H., Khanikaev, A. B. \& Wang, Z. Topologically protected elastic waves in phononic metamaterials. Nat. Commun. 6, 8682 (2015).

19. Yang, Z. et al. Topological acoustics. Phys. Rev. Lett. 114, 114301 (2015)

20. Ni, X. et al. Topologically protected one-way edge mode in networks of acoustic resonators with circulating air flow. New J. Phys. 17, 053016 (2015).

21. Khanikaev, A. B., Fleury, R., Mousavi, S. H. \& Alu, A. Topologically robust sound propagation in an angular-momentum-biased graphene-like resonator lattice. Nat. Commun. 6, 8260 (2015).

22. Fleury, R., Sounas, D. L., Sieck, C. F., Haberman, M. R. \& Alù, A. Sound isolation and giant linear nonreciprocity in a compact acoustic circulator. Science 343, 516-519 (2014)

23. Zhu, X.-F. et al. Topologically protected acoustic helical edge states and interface states in strongly coupled metamaterial ring lattices. Preprint at http://arXiv.org/abs/1508.06243 (2015).

24. Fleury, R., Khanikaev, A. \& Alu, A. Floquet topological insulators for sound. Nat. Commun. 7, 11744 (2016).

25. Xiao, M. et al. Geometric phase and band inversion in periodic acoustic systems. Nat. Phys. 11, 240-244 (2015).

26. Xiao, M., Chen, W.-J., He, W.-Y. \& Chan, C. T. Synthetic gauge flux and Weyl points in acoustic systems. Nat. Phys. 11, 920-924 (2015).

27. Chen, Z.-G. et al. Accidental degeneracy of double Dirac cones in a phononic crystal. Sci. Rep. 4, 4613 (2014).

28. Li, Y., Wu, Y. \& Mei, J. Double Dirac cones in phononic crystals. Appl. Phys. Lett. 105, 014107 (2014)

29. Ma, T., Khanikaev, A. B., Mousavi, S. H. \& Shvets, G. Guiding electromagnetic waves around sharp corners: topologically protected photonic transport in metawaveguides. Phys. Rev. Lett. 114, 127401 (2015).

30. He, C. et al. Photonic topological insulator with broken time-reversal symmetry. Proc. Natl Acad. Sci. USA 113, 4924-4928 (2016).

31. Sakoda, K. Double Dirac cones in triangular-lattice metamaterials. Opt. Express 20, 9925-9939 (2012)

32. Li, Y. \& Mei, J. Double Dirac cones in two-dimensional dielectric photonic crystals. Opt. Express 23, 12089-12099 (2015).

33. He, C. et al. Tunable one-way cross-waveguide splitter based on gyromagnetic photonic crystal. Appl. Phys. Lett. 96, 111111 (2010).

34. Fu, L. Topological crystalline insulators. Phys. Rev. Lett. 106, 106802 (2011)

35. He, C., Lu, M.-H., Wan, W.-W., Li, X.-F. \& Chen, Y.-F. Influence of boundary conditions on the one-way edge modes in two-dimensional magneto-optical photonic crystals. Solid State Commun. 150, 1976-1979 (2010). 


\section{Acknowledgements}

The work was jointly supported by the National Basic Research Program of China (Grant No. 2012CB921503, 2013CB632904 and 2013CB632702) and the National Nature Science Foundation of China (Grant No. 11134006, No. 11474158, and No. 11404164). M.-H.L. also acknowledges the support of the Natural Science Foundation of Jiangsu Province (BK20140019) and the support from the Academic Program Development of Jiangsu Higher Education (PAPD).

\section{Author contributions}

C.H., M.-H.L. and X.-P.L. conceived the idea. C.H. performed the numerical simulation and fabricated the samples. C.H., X.N. and H.G. carried out the experimental

measurements. All the authors contributed to discussion of the results and manuscript preparation. M.-H.L., X.-P.L. and Y.-F.C. supervised all aspects of this work and managed this project.

\section{Additional information}

Supplementary information is available in the online version of the paper. Reprints and permissions information is available online at www.nature.com/reprints.

Correspondence and requests for materials should be addressed to M.-H.L., X.-P.L. or Y.-F.C.

\section{Competing financial interests}

The authors declare no competing financial interests. 


\section{Methods}

Experiments. The phononic 'graphene' consists of commercial 304 stainless-steel rods with radii $r=0.3 \mathrm{~cm}$ and $r=0.45 \mathrm{~cm}$, arranged in air in graphene-like lattices. The radii tolerance of these steel rods is $\pm 0.01 \mathrm{~cm}$. The height of our phononic 'graphene' is chosen to be $20 \mathrm{~cm}$, roughly 10 times larger than the acoustic wavelength in air at $20 \mathrm{kHz}$ to make sure that two-dimensional approximation is applicable. Experiments are conducted with a large-area acoustic transducer. The transducer excites a roughly planar acoustic field, which then will be scattered at the input facet into the appropriate spin edge state according to the symmetry of the interface. A B\&K-4939-2670 microphone acts as a detector, which is placed $2 \mathrm{~cm}$ away from the output port with its response acquired and analysed in B\&K-3560-C. Acoustic input frequencies are swept from $18 \mathrm{kHz}$ to $22 \mathrm{kHz}$ with an increment of $0.01 \mathrm{kHz}$. The experimentally measured transmission spectra plotted in Figs $2 \mathrm{~d}$,e and $3 \mathrm{c}$, d are normalized to the acoustic wave transmission through the same distance in air. In theory, the transmission should be $100 \%(0 \mathrm{~dB})$ for the topological edge states with frequencies from $19.00 \mathrm{kHz}$ to $20.53 \mathrm{kHz}$. The deviation recorded in experiments (between $0 \mathrm{~dB}$ and $-5 \mathrm{~dB}$ ) is primarily due to the inefficient coupling into and out of the topological waveguides.

Simulations. Numerical investigations to calculate Figs $1 c, 2 b, c$ and $3 b$ in this letter are conducted using the acoustic mode of commercial FEM software (COMSOL MULTIPHYSICS)

Data availability. The data that support the plots within this paper and other findings of this study are available from the corresponding author on request. 